\title{
Performance Analysis of Energy Efficient Distributed Antenna Systems
}

\author{
Mansour M. Aldosari and Khairi Ashour Hamdi \\ School of Electrical and Electronic Engineering, \\ The University of Manchester, Manchester M13 9PL, U.K. \\ Email: mansour.aldosari-2@postgrad.manchester.ac.uk, k.hamdi@manchester.ac.uk.
}

\begin{abstract}
There is a huge demand for data traffic caused by increased usage of data-hungry applications on smart mobile devices. This has resulted into cellular network expansion and upgrade that have increased energy costs and generated environmental concerns. Distributed antenna systems (DAS) are applied to enhance the coverage of the cell via geographically distributed antennas elements (DAEs) which are connected to the base station that is located at the centre of the cell. In this paper, DAS is proposed as a network solution to fulfill increasing capacity demands while addressing the energy efficiency (EE) and environmental concerns associated with cellular network operation. Maximum Ratio Transmission (MRT) and Fractional Frequency Reuse (FFR) are applied to calculate the downlink ergodic spectral efficiency (SE), EE and energy consumption ratio (ECR). Nakagami-There is a huge demand for data traffic caused by increased usage of data-hungry applications on smart mobile devices. This has resulted into cellular network expansion and upgrade that have increased energy costs and generated environmental concerns. Distributed antenna systems (DAS) are applied to enhance the coverage of the cell via geographically distributed antennas elements (DAEs) which are connected to the base station that is located at the centre of the cell. In this paper, DAS is proposed as a network solution to fulfill increasing capacity demands while addressing the energy efficiency (EE) and environmental concerns associated with cellular network operation. Maximum Ratio Transmission (MRT) and Fractional Frequency Reuse (FFR) are applied to calculate the downlink ergodic spectral efficiency (SE), EE and energy consumption ratio (ECR). Nakagami- $m$ fading and log-normal shadowing are considered. Our results demonstrate that DAS using MRT and FFR increases the spectral and energy efficiency of the network compared to a basic cellular network system.

Key words - Ergodic Spectral Efficiency (ESE); Energy Efficiency (EE); Energy Consumption Ratio (ECR) ; Maximum Ratio Transmission (MRT); Fractional Frequency Reuse (FFR).
\end{abstract}

\section{INTRODUCTION}

The rapid revolution in smart phones and their applications is driving enhancement to the mobile communication networks infrastructure to fulfil the massive demands on the data rate. Capacity is one of the important performance measures for communications networks. Many researchers have applied different methods and techniques to increase the throughput of cellular communication networks; Distributed Antenna Systems (DAS) is currently one of the optimal solutions to increase the throughput [1] [2] and improve the coverage in dead spots, and [3] indoor (iDAS) [4] or outdoor (oDAS) communications can mitigate the problems of cell-edge coverage [5]. In DAS, remote antennas are spatially distributed within a structure or geographical area to reduce the distance between a remote antenna (RA) and a mobile terminal (MT), and thereby default enhance, modify and extend the coverage in the cell. DAS can be used in locations with higher populations such as stadiums, airports, large buildings, convention centres, and in areas experiencing bad coverage such as city centres with high rise buildings, etc.

DAS was proposed as a method to enhance the coverage of indoor wireless communications [4]. The ergodic capacity is determined under the influence of Rayleigh fading and lognormal shadowing by considering the signal to interference ratio (SIR) [1], and applies cooperative and non-cooperative RA. Authors in [3] investigated the downlink capacity for two different strategies - blanket transmission and selective diversity. In this paper, it has been shown that the capacity of the network can be increased by about two times due to the use of DAS, which leads to a reduction in other cell interference in a multi-cell environment. Authors in [2] uses fractional frequency reuse (FFR) and unity frequency reuse UFR, and applies coordinate multipoint (CoMP) cooperation to maximize cell throughput. [5] presents an improvement of the cell edge performance when using DAS, compared to a colocated antenna systems (CAS). Distributed antenna systems aid macrocell (MC) and femto cells (FCs) to coexist within the same area, and this is used to achieve high spectral efficiency (SE) for outdoor MC users (OMU) and indoor FC (IFC) users, using unity frequency reuse (UFR) and FFR [6]. Selection transmission (ST) is used in DAS to optimize the distributed antenna element (DAE) locations with or without the central antenna, and to maximize the expected signal-to-noise ratio (SNR) lower bound in each region [7]. In addition cooperative DAS is described by [8] for three different types of coverage depends on the MT location. Authors concludes that, at certain distances from the cell centre, cooperative DAS techniques improves the system capacity.

The main contributions of this paper are as follows:

- Derivation of new equations to calculate DAS downlink ergodic spectral efficiency using MRT. 


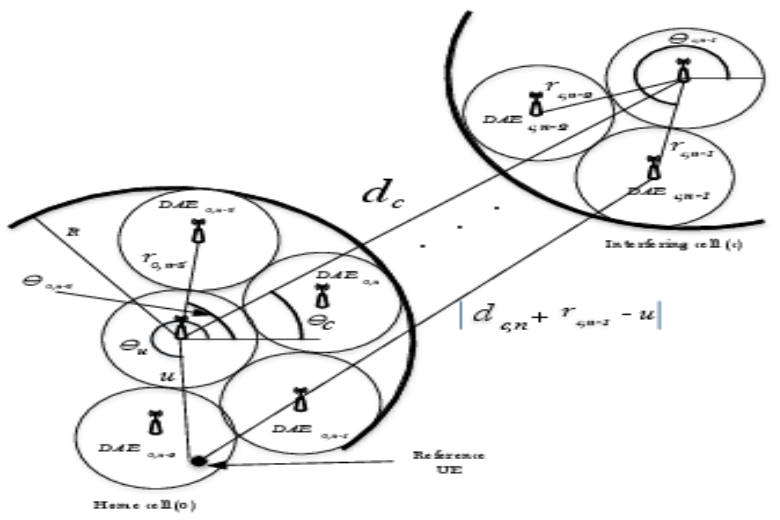

Figure 1. System model

- Calculation of the SE, EE and ECR of two DAS techniques and comparing them with basic model.

- Verification of the above results using a Monte Carlo simulation.

\section{System MOdEL}

In this paper, MRT method is applied and two techniques of DAS are investigated to calculate the ergodic SE of the reference MT to find which technique will provide better energy efficiency. A cluster of three-cell structure is proposed, where there are 7 DAEs in each cell, all connected to the the central BS. DAE $1, \mathrm{DAE}_{2}, \mathrm{DAE}_{3}, \ldots, \mathrm{DAE}_{\mathrm{N}}$ transmit an equal power signal. Fig. 1 shows the locations of DAEs in each cell relative to the centre of their home cells.

\section{A. Cellular Architecture}

Fractional Frequency Reuse: In this method, FFR is applied to calculate the ergodic spectral effeciency of MT in the cell.

In this case, it is assumed that the DAE in the cell centre uses a different frequency band $f_{1}$ while the other 6 DAEs use the same frequency band $f_{2}$. Therefore, all six DAEs which use the same frequency will cooperate to serve the MT in the same cell.

No Fractional Frequency Reuse: In this case, It is assumed that all DAE in the cell use same frequency band $f_{1}$. Therefore, all seven DAEs in the same cell will cooperate to serve the MT.

\section{B. Channel Model and Received Signal}

The received signal by the reference MT in the shell area is represented by

$$
\begin{aligned}
y= & \underbrace{\sqrt{P} x_{0} \sum_{n=1}^{N} \sqrt{G_{0, n}} h_{0, n} w_{0, n}}_{\text {Useful-signals }} \\
& +\underbrace{\sum_{c=1}^{C} \sqrt{P} x_{c} \sum_{n=1}^{N} \sqrt{G_{c, n}} h_{c, n} w_{c, n}}_{\text {Interfering-signals }}+n_{0}
\end{aligned}
$$

where $x_{0}$ is the transmitted signal by DAEs in the home cell and $x_{c}$ is the transmitted signal by $\mathrm{N}$ DAEs in the cell c. $h_{0, n}=\left\{h_{0,1}, h_{0,2}, \ldots h_{0,7}\right\}$ and $\left|h_{0, n}\right|^{2}$ is the channel gain of signal power received by the reference MT from all cooperative DAEs. $h_{c, n}=\left\{h_{1,1}, h_{1,2}, \ldots h_{6,7}\right\}$ and $\left|h_{c, n}\right|^{2}$ is the channel gain of the signals power received from interfering DAEs which use the same frequency in the other cells. In addition, $n_{0}$ is the additive white Gaussian noise (AWGN) at MT and satisfies $\mathbb{E}\left[\left|n_{0}\right|^{2}\right]=\sigma_{0}^{2}, P$ is the transmitted power and $\mathrm{N}$ is the number of proposed DAEs in each cell. The first part of (1) is the useful signal and the second and third parts are considered as interfering signals and noise,

where

$$
G_{c, n}=\left|d_{c, n}+r_{c, n}-u\right|^{-\beta} \xi_{c, n}
$$

is the large-scale signal level and $\xi_{c, n} \sim \log N\left(0, \sigma^{2}\right)$ is a random variable representing shadowing. Log-normal shadowing is assumed with $10 \log \xi_{c, n}$ a zero mean Gaussian with variance $\sigma^{2} . d_{c, n}$ is the locations of the interfering cells relative to the home cell and $u$ is the random position of the MT relative to it is home cell, and $\beta$ is the path loss exponent. Variable $r_{c, n}$ are the positions of the interfering $\mathrm{DAE}_{n}$ relative to the centre of it is home cell $c$, where $r_{c, n}=\left\{r e^{j \frac{2 \pi}{n}}, r e^{j \frac{2 \pi}{n-1}}, \ldots r e^{j 2 \pi}\right\}$ and $d_{c, n}=\left\{d_{c} e^{j \frac{2 \pi}{n}}, d_{c} e^{j \frac{2 \pi}{n-1}}, \ldots d_{c} e^{j 2 \pi}\right\}, d_{c}$ is the frequency reuse distance as shown in Fig. 1. The distance between the MT and interfering DAEs can be calculated using Euler's formula, and the large scale signal level is expressed as

$$
\begin{aligned}
G_{c, n} & =\mid d_{c}\left(\cos \left(\theta_{c}\right)+j \sin \left(\theta_{c}\right)\right) \\
& +r\left(\cos \left(\theta_{c, n}\right)+j \sin \left(\theta_{c, n}\right)\right)-\left.u\right|^{-\beta} \xi_{c, n}
\end{aligned}
$$

where $\theta_{c}$ is the angles of the interfering cells relative to the home cell and $\theta_{c, n}$ is the angles of the interfering $\mathrm{DAE}_{c, n}$ relative to the centre of it is home cell.

In the home cell

$$
\begin{aligned}
G_{0, n} & =\left|d_{0, n}+r_{0, n}-u\right|^{-\beta} \xi_{c, n} \\
& =\left|r\left(\cos \left(\theta_{0, n}\right)+j \sin \left(\theta_{0, n}\right)\right)-u\right|^{-\beta} \xi_{0, n}
\end{aligned}
$$

where $d_{0, n}$ is equal to zero at the home cell and $\theta_{0, n}$ is the angles of the $\mathrm{DAE}_{n}$ relative to the centre of it is home cell .

In the following expressions $d_{c, n}, r_{0, n}$ and $r_{c, n}$ notations will be used to make the equations shorter.

In MRT

$$
w_{c, n}=\frac{\sqrt{G_{c, n}} h_{c, n}^{*}}{\sqrt{\sum_{n=1}^{N} G_{c, n}\left|h_{c, n}\right|^{2}}}
$$

Therefore, for the sake of spectral efficiency (SE) analysis, it is assumed that $x_{0}$ and $x_{c}$ are Gaussian and the signal to interference plus noise ratio (SINR) can be written as

$$
\mathrm{SINR}=\frac{\sum_{n=1}^{N} G_{0, n}\left|h_{0, n}\right|^{2}}{\sum_{c=1}^{C}\left|\sum_{n=1}^{N} G_{c, n} h_{c, n} w_{c, n}\right|^{2}+1 / \rho}
$$

where $\rho=\frac{P}{\sigma_{0}^{2}}$ is the signal-to-noise ratio (SNR) at the cell boundary (when the distance is normalized and the cell radius is unity) 


\section{SPECTRAL EFFICIENCY}

The ergodic (average) spectral efficiency achieved by an arbitrary user can be estimated by using the average of the Shannon capacity formula[9]:

$$
\begin{aligned}
& \mathrm{C}=\mathbb{E}\left[\log _{2}(1+\mathrm{SINR})\right] \\
& =\mathbb{E}\left[\log _{2}\left(1+\frac{\sum_{n=1}^{N} G_{0, n}\left|h_{0, n}\right|^{2}}{\sum_{c=1}^{C}\left|\sum_{n=1}^{N} G_{c, n} h_{c, n} w_{c, n}\right|^{2}+1 / \rho}\right)\right]
\end{aligned}
$$$$
[\mathrm{b} / \mathrm{s} / \mathrm{Hz}]
$$

In order to evaluate the average, Cauchy-Schwarz inequality is firstly invoke:

$$
\begin{aligned}
\left|\sum_{n=1}^{N} G_{c, n} h_{c, n} w_{c, n}\right|^{2} & \leq\left(\sum_{n=1}^{N} G_{c, n}\left|h_{c, n}\right|^{2}\right)\left(\sum_{n=1}^{N}\left|w_{c, n}\right|^{2}\right) \\
& =\sum_{n=1}^{N} G_{c, n}\left|h_{c, n}\right|^{2}
\end{aligned}
$$

This is used to rewrite (7) as follows

$$
\mathrm{C}(u, \theta)=\mathbb{E}\left[\log _{2}\left(1+\frac{\sum_{n=1}^{N} G_{0, n}\left|h_{0, n}\right|^{2}}{\sum_{c=1}^{C} \sum_{n=1}^{N} G_{c, n}\left|h_{c, n}\right|^{2}+1 / \rho}\right) \mid u, \theta\right]
$$

The expectation in (9) is to be applied with respect to the $\mathrm{N}+\mathrm{N} \times \mathrm{C}$ non-negative random variables. Classical methods employed to evaluate such an average require at least 49 numerical integrations, which will make the process very complicated. In order to reduce the computational complexity of (7), a non-direct method is invoked in the following, which greatly simplifies the required computation.

Notations: $\gamma_{0, n}=G_{0, n}\left|h_{0, n}\right|^{2}=\left|r_{0, n}-u\right|^{-\beta} \xi_{0, n}\left|h_{0, n}\right|^{2}$ and $\delta_{c, n}=G_{c, n}\left|h_{c, n}\right|^{2}=\left|d_{c, n}+r_{c, n}-u\right|^{-\beta} \xi_{c, n}\left|h_{c, n}\right|^{2}$ are used in the following eqations.

Therefore,

$$
\mathrm{C}(u, \theta)=\mathbb{E}\left[\log _{2}\left(1+\frac{\sum_{n=1}^{N} \gamma_{0, n}}{\sum_{c=1}^{C} \sum_{n=1}^{N} \delta_{c, n}+1 / \rho}\right)\right]
$$

\section{Lemma 1:}

From [10]:

$$
\ln (1+t)=\int_{0}^{\infty} \frac{1}{s}\left(1-e^{-s t}\right) e^{-s} \mathrm{~d} s, \quad t \geq 0
$$

Proof of Lemma 1: The following proof is based on [10, Eq. 6]:

Let $t=\frac{\sum_{n=1}^{N} \gamma_{0, n}}{\sum_{c=1}^{C} \sum_{n=1}^{N} \delta_{c, n}+1 / \rho}$ in (10). Then

$$
\begin{gathered}
\ln \left(1+\frac{\sum_{n=1}^{N} \gamma_{0, n}}{\sum_{c=1}^{C} \sum_{n=1}^{N} \delta_{c, n}+1 / \rho}\right) \\
=\int_{0}^{\infty} \frac{1}{s}\left(1-e^{-s \frac{\sum_{n=1}^{N} \gamma_{0, n}}{\sum_{c=1}^{C} \sum_{n=1}^{N} \delta_{c, n}+1 / \rho}}\right) e^{-s} \mathrm{~d} s .
\end{gathered}
$$

Substitute $s=z\left(\sum_{c=1}^{C} \sum_{n=1}^{N} \delta_{c, n}+1 / \rho\right)$, where $\mathrm{d} s=$ $\left(\sum_{c=1}^{C} \sum_{n=1}^{N} \delta_{c, n}+1 / \rho\right) \mathrm{d} z$. Therefore, from (12) we obtain

$$
\begin{aligned}
& \ln \left(1+\frac{\sum_{n=1}^{N} \gamma_{0, n}}{\sum_{c=1}^{C} \sum_{n=1}^{N} \delta_{c, n}+1 / \rho}\right) \\
&=\int_{0}^{\infty} \frac{1}{z}\left(1-e^{-z \sum_{n=1}^{N} \gamma_{0, n}}\right) \\
& \quad \times e^{-z \sum_{c=1}^{C} \sum_{n=1}^{N} \delta_{c, n}} e^{-\frac{z}{\rho}} \mathrm{d} z .
\end{aligned}
$$

Therefor, from (10) and (13), the spectral efficiency can be calculated as

$\mathbb{E}\left[\log _{2}\left(1+\frac{\sum_{n=1}^{N} \gamma_{0, n}}{\sum_{c=1}^{C} \sum_{n=1}^{N} \delta_{c, n}+1 / \rho}\right) \mid r, \theta\right]$

$=\mathbb{E}\left[\log _{2}(e) \int_{0}^{\infty} \frac{1}{z}\left(1-e^{-z \sum_{n=1}^{N} \gamma_{0, n}}\right) e^{-z\left(\sum_{c=1}^{C} \sum_{n=1}^{N} \delta_{c, n}+1 / \rho\right)} \mathrm{d} z\right]$

$=\mathbb{E}\left[\log _{2}(e) \int_{0}^{\infty} \frac{1}{z}\left(1-e^{-z \sum_{n=1}^{N} \gamma_{0, n}}\right) e^{-z \sum_{c=1}^{C} \sum_{n=1}^{N} \delta_{c, n}} e^{-\frac{z}{\rho}} \mathrm{d} z\right]$

$=\log _{2}(e) \int_{0}^{\infty} \frac{1}{z}\left(1-\mathbb{E}\left[e^{-z \sum_{n=1}^{N} \gamma_{0, n}}\right]\right)$

$\times \mathbb{E}\left[e^{-z \sum_{c=1}^{C} \sum_{n=1}^{N} \delta_{c, n}}\right] e^{-\frac{z}{\rho}} \mathrm{d} z$

$=\log _{2}(e) \int_{0}^{\infty} \frac{1}{z}\left(1-\mathscr{M}_{0}(z, u)\right) \prod_{c=1}^{C} \mathscr{M}_{c}(z, u) e^{-\frac{z}{\rho}} \mathrm{d} z$

It is possiple to represent the averages $\mathbb{E}\left[e^{-z \sum_{n=1}^{N} \gamma_{0, n}}\right]$ and $\mathbb{E}\left[e^{-z \sum_{c=1}^{C} \sum_{n=1}^{N} \delta_{c, n}}\right]$ by the moment generating function $\mathscr{M}_{0}(z, u)$ and $\mathscr{M}_{c}(z, u)$. Where

$$
\mathscr{M}_{0}(z, u)=\prod_{i=1}^{N} w_{0}\left(z\left|r_{0, n}-u\right|^{-\beta}\right)
$$

and

$$
\begin{gathered}
w_{0}(z)=\mathbb{E}\left[e^{-z \xi_{0, n}\left|h_{0, n}\right|^{2}}\right] \\
\mathscr{M}_{c}(z, u)=\prod_{i=1}^{N} w_{c}\left(z\left|d_{c}+r_{c, n}-u\right|^{-\beta}\right)
\end{gathered}
$$

and

$$
w_{c}(z)=\mathbb{E}\left[e^{-z \xi_{c, n}\left|h_{c, n}\right|^{2}}\right]
$$

To find the average of $w_{0}(z)$ and $w_{c}(z)$ where $\xi_{0, n}$ and $\xi_{c, n}$ are log-normal distributed random variable $(\mathrm{RV})$, composite Nakagami and Log-normal shadowing are applied [11]. The pdf of the composite gamma/log-normal distribution is

$$
\begin{aligned}
p_{x}(x) & =\int \frac{m^{m} x^{m-1}}{\psi^{m} \Gamma(m)} \exp \left(-\frac{m x}{\psi}\right) \times \frac{\varphi}{\sqrt{2 \pi \sigma^{2}}} \\
& \times \exp \left(\frac{-\left(10 \log _{10} \psi-\mu\right)^{2}}{2 \sigma^{2}}\right) \mathrm{d} \psi
\end{aligned}
$$

where $m$ is the Gamma distribution's shape parameter, and $\varphi=10(\ln 10)^{-1}$. 
The MGF of $\xi_{0, n}$ and $\xi_{c, n}$ can be derived as follows:

$$
w(z)=\int_{0}^{\infty} \exp ^{-x z} p_{x}(x) \mathrm{d} x
$$

Therefore, from equations (19) and (20)

$$
\begin{aligned}
w(z) & =\int_{0}^{\infty}\left(1+z \frac{\psi}{m}\right)^{-m} \times \frac{10(\ln 10)^{-1}}{\sqrt{2 \pi} \sigma} \\
& \times \exp \left(\frac{-\left(10 \log _{10} \psi-\mu\right)^{2}}{2 \sigma^{2}}\right) \mathrm{d} \psi
\end{aligned}
$$

we assume $t=\frac{10 \log _{10} \psi-\mu}{\sqrt{2} \sigma}$ and $\psi=10^{\frac{\sqrt{2} \sigma t+\mu}{10}}$ where $\frac{\mathrm{d} \psi}{\mathrm{d} t}=$ $\psi \frac{\sigma \ln (10)}{5 \sqrt{2}}$ which means $\mathrm{d} \psi=\psi \frac{\sigma \ln (10)}{\sqrt{50}} \mathrm{~d} t$. Therefore,

$$
w(z)=\int_{-\infty}^{\infty} \frac{1}{\sqrt{\pi}}\left(1+z \frac{10^{\frac{t \sqrt{2} \sigma+\mu}{10}}}{m}\right)^{-m} \times \exp \left(-t^{2}\right) \mathrm{d} t
$$

To calculate the above formula, apply the GaussHermite quadrature as in [[12], 25.4.46] which represents $\int_{-\infty}^{\infty} \exp \left(-x^{2}\right) f(x)=\sum_{l=1}^{L} w_{l} f\left(x_{l}\right)$, where $w_{l}$ and $f\left(x_{l}\right)$ are the weight and abscissas factors of $\mathrm{L}$ values according to [[12], table 25.10] which provides the following:

$$
w(z, u) \backsim \sum_{l=1}^{L} \frac{w_{l}}{\sqrt{\pi}}\left(1+z \frac{[d(u, \theta)]^{-\beta} 10^{\frac{\sqrt{2} \sigma x_{l}+\mu}{10}}}{m}\right)^{-m}
$$

From the above, it is possible to calculate the $\operatorname{MGF} \mathscr{M}_{0}(z, u)$ of the useful signals as

$\mathscr{M}_{0}(z, u) \simeq \prod_{n=1}^{N} \sum_{l=1}^{L} \frac{w_{l}}{\sqrt{\pi}}\left(1+z \frac{\left|r_{0, n}-u\right|^{-\beta} 10^{\frac{\sqrt{2} \sigma x_{l}+\mu}{10}}}{m}\right)^{-m}$,

and the MGF $\mathscr{M}_{c}(z, u)$ of the interfering signals is

$\mathscr{M}_{c}(z, u) \simeq \prod_{c=1}^{C} \prod_{n=1}^{N} \sum_{l=1}^{L} \frac{w_{l}}{\sqrt{\pi}}\left(1+z \frac{\left|d_{c, n}+r_{c, n}-u\right|^{-\beta} 10^{\frac{\sqrt{2} \sigma x_{l}+\mu}{10}}}{m}\right)_{(25)}^{-m}$

Therefore, the ergodic spectral efficiency is written as

$$
\mathrm{C}(u, \theta)=\int_{0}^{\infty} \int_{0}^{R} \int_{0}^{2 \pi} \frac{1}{z}
$$

$\times\left(1-\prod_{n=1}^{N} \sum_{l=1}^{L} \frac{w_{l}}{\sqrt{\pi}}\left(1+z \frac{\left|r_{0, n}-u\right|^{-\beta} 10^{\frac{\sqrt{2} \sigma x_{l}+\mu}{10}}}{m}\right)^{-m}\right)$

$\times \prod_{c=1}^{C} \prod_{n=1}^{N} \sum_{l=1}^{L} \frac{w_{l}}{\sqrt{\pi}}\left(1+z \frac{\left|d_{c, n}+r_{c, n}-u\right|^{-\beta} 10^{\frac{\sqrt{2} \sigma x_{l}+\mu}{10}}}{m}\right)^{-m}$

$$
\times \frac{r}{\pi R^{2}} e^{-\frac{z}{\rho}} \log _{2}(\mathrm{e}) \mathrm{d} \theta \mathrm{d} u \mathrm{~d} z
$$

Ergodic spectral efficiency of the MT against the distance from the BS at the centre toward the edge of the cell is expressed as below

$$
\begin{aligned}
& \mathrm{C}(u, \theta)=\int_{0}^{\infty} \frac{1}{z} \\
& \times\left(1-\prod_{n=1}^{N} \sum_{l=1}^{L} \frac{w_{l}}{\sqrt{\pi}}\left(1+z \frac{\left|r_{0, n}-u\right|^{-\beta} 10^{\frac{\sqrt{2} \sigma x_{l}+\mu}{10}}}{m}\right)^{-m}\right) \\
& \times \prod_{c=1}^{C} \prod_{n=1}^{N} \sum_{l=1}^{L} \frac{w_{l}}{\sqrt{\pi}}\left(1+z \frac{\left|d_{c, n}+r_{c, n}-u\right|^{-\beta} 10^{\frac{\sqrt{2} \sigma x_{l}+\mu}{10}}}{m}\right)^{-m} \\
& \times e^{-\frac{z}{\rho}} \log _{2}(e) \mathrm{d} z
\end{aligned}
$$

\section{DAS ENERGY EFFICIENCY}

The power consumed by DAS $\mathrm{P}_{D A S}$ can by classified in two major parts: power consumed by power amplifier (PA) and the power consumed by other circuit components as [13]

$$
\mathrm{P}_{D A S}=\mathrm{P}_{P A}+\mathrm{P}_{c}
$$

where $\mathrm{P}_{c}$ is the power consumed by circuit components and $P_{P A}$ is the power consumed by power amplifier which can be expressed by

$$
\mathrm{P}_{P A}=(1+\tau) \mathrm{P}_{t}
$$

where $\tau=\frac{\varsigma}{\wp}-1, \varsigma$ is the peak-to-average ratio (PAR) which is defined as the ratio between the average and maximal power, $\wp$ is the radio frequency power amplifier drain efficiency and $P_{t}$ is the transmit power consumption.

$$
\mathrm{P}_{c}=\mathrm{P}_{s}+\zeta \mathrm{C}(u, \theta)
$$

where $\mathrm{P}_{s}$ is a static power consumption, $\zeta$ is a constant representing dynamic power consumption per throughput and $\mathrm{C}(u, \theta)$ is the spectral efficiency as in equation (27).

Therefore, the energy efficiency (EE) can be estimated as follows [14]

$$
\mathrm{EE}=\frac{\mathrm{WC}(u, \theta)}{\mathrm{P}_{D A S}} \quad[\text { bits/Joule }]
$$

where $\mathrm{W}$ represents the cell bandwidth.

The energy consumption ratio [15] can be calculated using the following expression

$$
\mathrm{ECR}=\frac{\mathrm{P}_{D A S}}{\mathrm{WC}(u, \theta)} \quad[\text { Joule } / \mathrm{bit}]
$$

\section{NUMERICAL RESULTS}

From the figures, it can be concluded that using DAS improves the SE of the network compared to the basic model as the BS-MT separation distance increases. This helps to overcome poor coverage at the edge of the cell especially in dense urban areas. Fig. 2 shows that SE is increased by $86 \%$ when DAS is applied compared to the basic model. Conversely, this figure shows a massive degradation in SE of the basic model when the MT moves further away from the centre of the cell. In the same figure, SE is is degraded when FFR is applied due to the decrease in the frequency reuse 


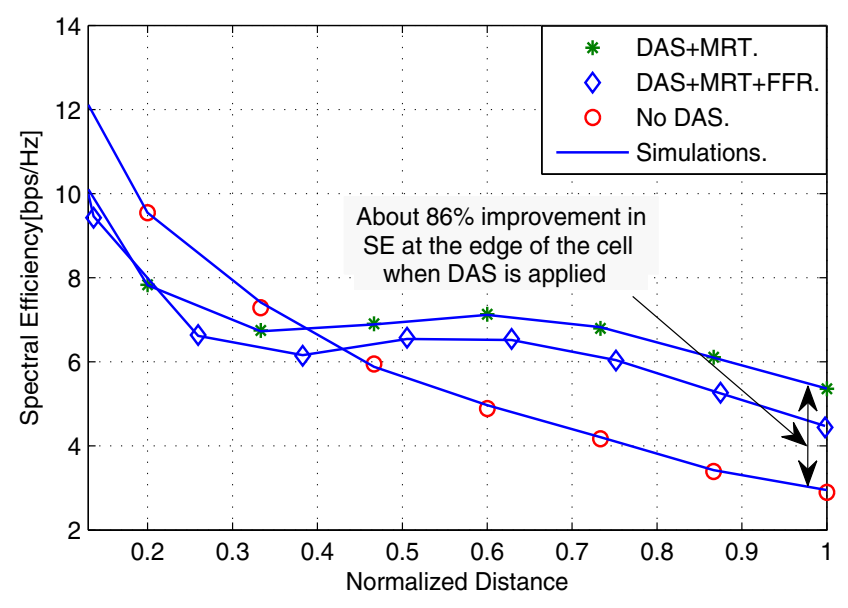

Figure 2. Spectral efficiency comparison at SNR $=10 \mathrm{~dB}$ using FFR with and without using DAS.

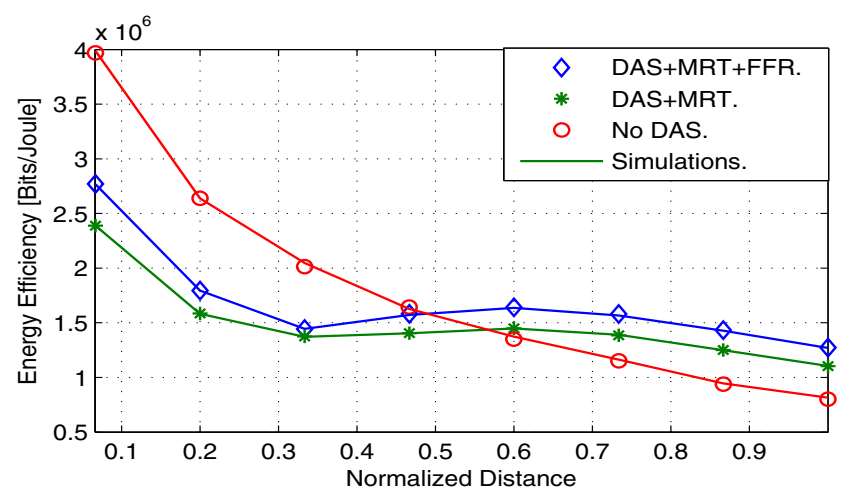

Figure 3. Energy efficiency comparison at $\mathrm{SNR}=10 \mathrm{~dB}$ using FFR with and without using DAS.

distance. Fig. 3 shows that applying DAS gives about $43 \%$ improvement in the EE at the cell edge compared to the basic model. Moreover, EE is improved by about $62 \%$ when FFR is applied with DAS compared to the basic model. Finally, Fig. 4 shows that there is about $27 \%$ enhancement in the ECR at the cell edge when DAS is applied. Also, ECR is improved by about $36 \%$ at the same distance when FFR is applied with DAS compared to the basic system.

\section{CONCLUSION}

In this paper, the potential of DAS to enhance the SE of a cellular network is invistigated. From the numerical results above, it can be concluded that DAS is one of the options available to cellular network operators and designers to increase the throughput of cellular networks and enhance the coverage of the network especially in the cell edge region where coverage is normally poor. Furthermore, DAS with FFR gives a better energy performance as measured using $\mathrm{EE}$ and ECR compared to the basic cellular network model.

\section{REFERENCES}

[1] J. Park, E. Song, and W. Sung, "Capacity analysis for distributed antenna systems using cooperative transmission schemes in fading channels,"

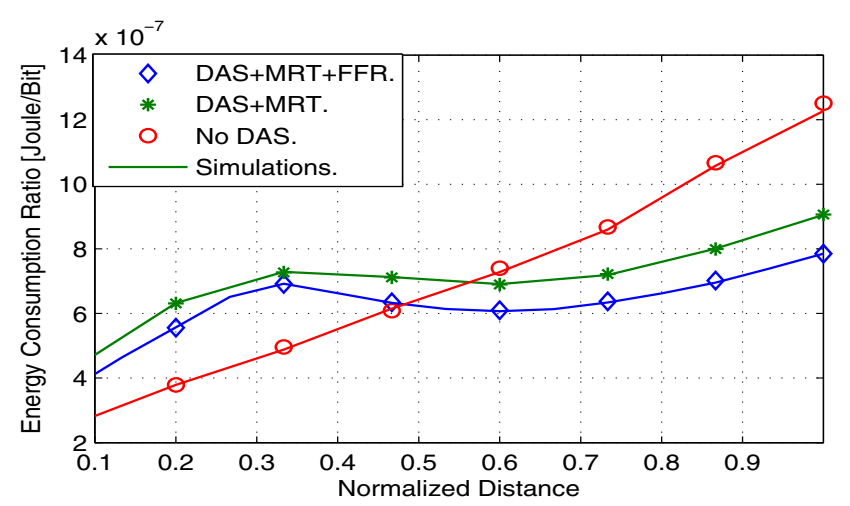

Figure 4. Energy cosumption ratio comparison at $\mathrm{SNR}=10 \mathrm{~dB}$ using FFR with and without using DAS.

[2] J. Zhang, R. Zhang, G. Li, and L. Hanzo, "Distributed antenna systems in fractional-frequency-reuse-aided cellular networks," Vehicular Technology, IEEE Transactions on, vol. 62, no. 3, pp. 1340-1349, 2013.

[3] W. Choi and J. Andrews, "Downlink performance and capacity of distributed antenna systems in a multicell environment," Wireless Communications, IEEE Transactions on, vol. 6, no. 1, pp. 69-73, Jan 2007.

[4] A. A. Saleh, A. Rustako, and R. Roman, "Distributed antennas for indoor radio communications," Communications, IEEE Transactions on, vol. 35, no. 12, pp. 1245-1251, 1987.

[5] X. You, D. Wang, P. Zhu, and B. Sheng, "Cell edge performance of cellular mobile systems," Selected Areas in Communications, IEEE Journal on, vol. 29, no. 6, pp. 1139-1150, June 2011.

[6] J. Zhang, F. Jin, R. Zhang, G. Li, and L. Hanzo, "Analysis and design of distributed antenna-aided twin-layer femto- and macrocell networks relying on fractional frequency reuse," Vehicular Technology, IEEE Transactions on, vol. 63, no. 2, pp. 763-774, Feb 2014.

[7] E. Park and I. Lee, "Antenna placement for downlink distributed antenna systems with selection transmission," in Vehicular Technology Conference (VTC Spring), 2011 IEEE 73rd, May 2011, pp. 1-5.

[8] X. Zhao and X. Yang, "Downlink ergodic capacity analysis for wireless networks with cooperative distributed antenna systems," in Communications (ICC), 2012 IEEE International Conference on, June 2012, pp. 5911-5915.

[9] K. Hamdi, "A useful lemma for capacity analysis of fading interference channels," Communications, IEEE Transactions on, vol. 58, no. 2, pp. 411-416, 2010.

[10] K. A. Hamdi, "Capacity of mrc on correlated rician fading channels," Communications, IEEE Transactions on, vol. 56, no. 5, pp. 708-711, 2008.

[11] M.-S. A. Marvin K. Simon, Digital Communication over Fading Channels. Wiley-IEEE Press, January 2005.

[12] I. Abramowitz, M.; Stegun, Handbook of Mathematical Functions with Formulas, Graphs, and Mathematical Tables (Partially Mathcadenabled). U.S. Department of Commerce, NIST, 2002-03-27.

[13] C. He, B. Sheng, P. Zhu, X. You, and G. Li, "Energy- and spectralefficiency tradeoff for distributed antenna systems with proportional fairness," Selected Areas in Communications, IEEE Journal on, vol. 31, no. 5, pp. 894-902, May 2013.

[14] L. Deng, Y. Rui, P. Cheng, J. Zhang, Q. Zhang, and M. Li, "A unified energy efficiency and spectral efficiency tradeoff metric in wireless networks," Communications Letters, IEEE, vol. 17, no. 1, pp. 55-58, 2013.

[15] B. Badic, T. O'Farrrell, P. Loskot, and J. He, "Energy efficient radio access architectures for green radio: Large versus small cell size deployment," in Vehicular Technology Conference Fall (VTC 2009-Fall), 